\title{
Rotational Roadmapping: A New Image-Based Navigation Technique for the Interventional Room
}

\author{
Markus Kukuk ${ }^{1,2}$ and Sandy Napel ${ }^{2}$ \\ ${ }^{1}$ Siemens Medical Solutions, Forchheim, Germany \\ ${ }^{2}$ Stanford University, Department of Radiology, USA \\ kukuk@stanford.edu
}

\begin{abstract}
For decades, conventional 2D-roadmaping has been the method of choice for image-based guidewire navigation during endovascular procedures. Only recently have 3D-roadmapping techniques become available that are based on the acquisition and reconstruction of a 3D image of the vascular tree. In this paper, we present a new image-based navigation technique called RoRo (Rotational Roadmapping) that eliminates the guess-work inherent to the conventional 2D method, but does not require a 3D image. Our preliminary clinical results show that there are situations in which RoRo is preferred over the existing two methods, thus demonstrating potential for filling a clinical niche and complementing the spectrum of available navigation tools.
\end{abstract}

\section{Introduction}

The number and breadth of minimally invasive, image-guided therapies is ever increasing. Of particular interest are endovascular procedures, which allow minimally invasive access to all areas of the human body through the vascular system as, for example angioplasty, vascular stenting, embolization, chemoembolization, thrombolysis and TIPS (Transjugular Intrahepatic Portosystemic Shunt). These procedures are typically performed in an interventional room using C-arm based X-ray imaging (see Fig. 1), together with the selective injection of contrast material for blood vessel opacification. Common to all endovascular procedures is the navigation of a guidewire or catheter through the vasculature to a target site. Depending on the degree of tortuousity and structural complexity of the vascular tree, especially in diseased vasculature, guidance (image- or sensor-based [1]) is often required for targeted steering.

Essentially unchanged from its first introduction in the early 1980s, a technique called 2D-roadmapping [2] has long become clinical routine for image-based guidewire navigation. The basic idea is to acquire an image of the vasculature of interest and to store it as a "roadmap" image. Then, the guidewire or other instrument as shown under live fluoroscopy is continuously superimposed onto the roadmap image, thus visualizing the instrument with respect to the vasculature. For acquiring the roadmap image, the interventionalist estimates how to position the $\mathrm{C}$-arm for finding a suitable working view, which is considered ideal if it shows the vessel bifurcation to be negotiated perpendicular to the viewing direction, thus eliminating self occlusion. However, the vessel tree is visible only after the injection of contrast media, which 

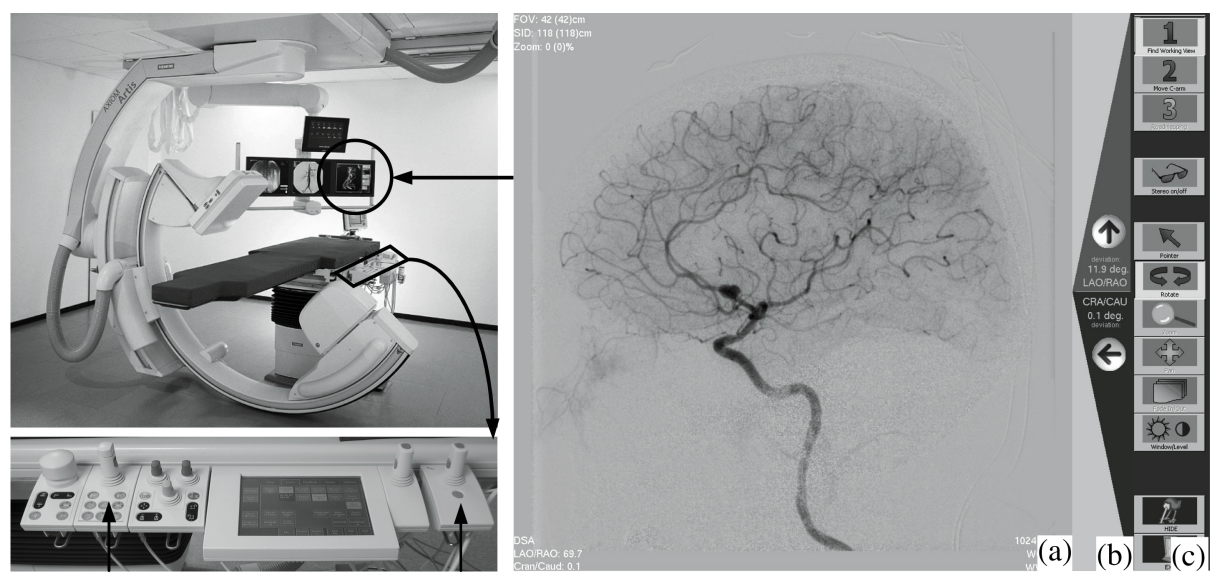

Fig. 1. Left: C-arm system "Siemens Axiom Artis $d$ TA" (top) and its table side controls (bottom). Arrows indicate the C-arm joystick (left) and RoRo joystick (right). Right: RoRo screen, consisting of three columns: (a) image panel, (b) alignment signal, (c) control panel.

may or may not reveal the desired viewing angle. During the course of a lengthy intervention, the individual injections of contrast material, which is toxic in large doses, can add up to a significant amount due to the trial-and-error nature of the approach.

Only recently, a new image-based navigation technique has been introduced: 3Droadmapping [3]. This technique is based on the acquisition and reconstruction of a 3D image (C-arm CT, CTA, MRA) of the vessel tree, which then serves as the roadmap image. CTA and MRA are acquired during an intra-venous contrast injection and require registration with the $\mathrm{C}$-arm system (2D/3D registration [4]). C-arm CT images, acquired during a selective, intra-arterial injection during the procedure are obtained from a rotational acquisition of projection images and the subsequent application of a cone-beam reconstruction method [5]. Following a one-time calibration step, one can correctly render an image of the 3D volume as would be "seen" by any given $\mathrm{C}$-arm configuration. In other words, roadmap images are available from any angle, thus eliminating the guess-work in finding an ideal working view. In situations where a 3D angiographic acquisition is routinely performed for diagnostic purposes, 3D-roadmapping appears to be the perfect navigation tool at no extra "cost" regarding dose or contrast agent.

However, there are clinical situations in which a 3D acquisition for the purpose of navigation is either not justified or technically challenging. The following represents a list of situations that may cause reconstruction artifacts and may therefore result in compromised image quality: cardiac and respiratory motion during acquisition, inhomogenous flow of contrast material due to cardiac pulsation, high velocity flow of contrast material, photon starvation along the shoulder axis and in the presence of indwelling metal such as coils and stents. Furthermore, image quality is directly related to the number of acquired projections and therefore a function of volume of contrast media injected.

In this paper we present a new image-based navigation technique that fits technically and clinically between the described 2D- and 3D roadmapping techniques: 
Rotational Roadmapping (RoRo). RoRo is based on a single rotational acquisition of multiple views (2.5D-roadmapping). Because 3D reconstructions are not performed, acquisitions of any length and any number of projections are possible. Instead, the projections are directly used for roadmap navigation.

RoRo can be regarded as a natural extension of the conventional 2D roadmapping technique, as it allows rotating the $\mathrm{C}$-arm during contrast injection, instead of keeping it stationary. Thus, more information is acquired per unit of contrast agent, producing a "rotatable roadmap" that shows the vessel tree from multiple views. After the acquisition, each projection of the rotatable roadmap can be selected to serve as a roadmap image for classic 2D-roadmapping navigation. The same rotatable roadmap can be used again and again to find the ideal working view for each segment of the vessel tree, thus rendering the injection of additional contrast media unnecessary. Furthermore, the vessel trajectories can be viewed in 3D, using stereographic visualization (3D glasses) using suitably selected pairs of views, separated by a small angle [6].

We have developed a research prototype that implements the RoRo approach, with software and hardware fully integrated into a C-arm system (Axiom Artis $d \mathrm{TA}, d \mathrm{BA}$, Siemens Medical Solutions) that can be controlled during an intervention from the patient table side (see Fig. 1). We present first clinical results that demonstrate RoRo's potential for filling the gap between the existing methods.

\section{Material and Methods}

Although RoRo's principal idea is straightforward, several challenges need to be addressed in order to provide a solution that meets the high demands of clinical, intraoperative software: seamless workflow integration, accurate $\mathrm{C}$-arm/image alignment and vessel tree/instrument visualization.

\subsection{Clinical Workflow}

1. Image acquisition (not part of RoRo)

2. Image transfer to RoRo

3. RoRo Phase 1: Find (suitable) working view

4. RoRo Phase 2: Align C-arm with selected working view

5. RoRo Phase 3: Roadmapping (Instrument guidance using selected working view)

6. If a different working view is needed, return to RoRo Phase 1

Image acquisition consists of a conventional rotational acquisition of any length and angular increment. For example, in case of a rotational DSA (Digital Subtraction Angiography) acquisition, two identical rotational runs are performed: the first without (mask run) and the second with the injection of contrast media (fill run). After acquisition, the study consisting of two runs of 2D projections is sent to the RoRo application by means of a DICOM transfer.

Upon completion of the image transfer, RoRo computes a third "DSA run," by subtracting corresponding fill from mask images. RoRo then automatically enters phase 1 by displaying the DSA run as a "rotatable roadmap." For finding a suitable working view, the $\mathrm{C}$-arm and the rotatable roadmap are linked to each other in two 


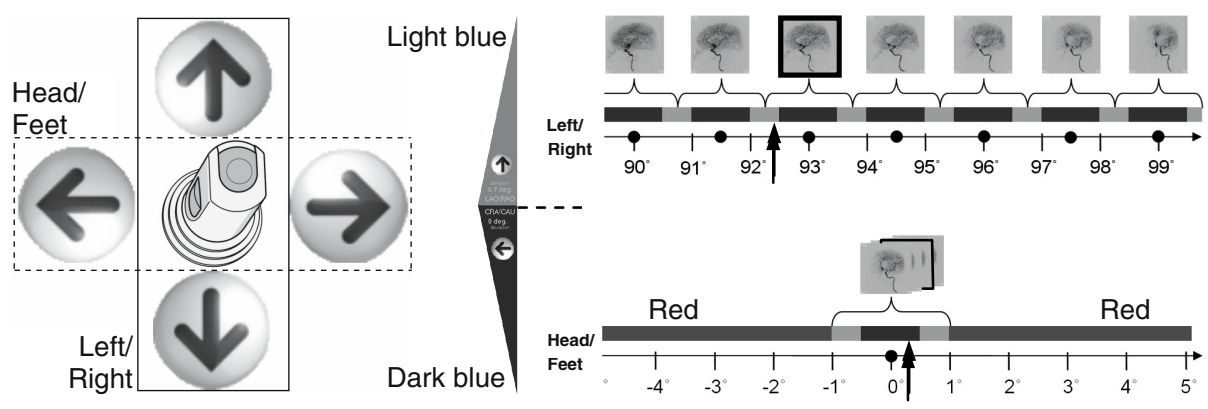

Fig. 2. Interactive $\mathrm{C}$-arm/image alignment. Left: $\mathrm{C}$-arm joystick; Center: Alignment signal. Right: C-arm's Left/Right (top) and Head/Feet (bottom) plane, indicating the current C-arm position by an arrow and the currently displayed image by a box. Images were acquired every $1.5^{\circ}$ (dots) along the Left/Right plane at $0^{\circ} \mathrm{Head} /$ Feet angulation. The deviation between the $\mathrm{C}$-arm position and the currently displayed image is indicated by the alignment signal. The top half shows the alignment for the Left/Right plane to be within $1.0^{\circ}$ (light blue) while the bottom half shows the alignment for the Head/Feet plane to be within $0.5^{\circ}$ (dark blue). The two arrows in the alignment signal indicate the direction in which to move the joystick to improve alignment for the respective plane: "upward" and "left" will move the C-arm closer to $93^{\circ} \mathrm{L} / \mathrm{R}$ and $0^{\circ} \mathrm{H} / \mathrm{F}$.

ways, as described in the next section. At this point, zoom formats and SID (Source Image Distance) can be adjusted as needed.

Once the C-arm and the selected working view are aligned, RoRo enters Phase 3 "roadmapping." By pressing the fluoro footswitch, live fluoroscopic images of the instruments being guided are acquired and overlaid onto the selected view of the vessel tree, similar to the classic 2D roadmapping technique. At any time during Phase 3 , the user can return to Phase 1 for finding a new working view. This is done either by moving the $\mathrm{C}$-arm into a new position (roadmap follows $\mathrm{C}$-arm), or by using the RoRo-joystick to rotate the roadmap (C-arm follows roadmap). The RoRo joystick is sterilized together with the standard controls by draping them in clear plastic sheets.

\subsection{C-Arm/Image Alignment}

One principal challenge with the RoRo approach is to provide for a fast and accurate alignment of the $\mathrm{C}$-arm with the currently displayed working view. After image acquisition, vessel images are only available at discrete points along the acquisition trajectory (see Fig. 2, right), while the C-arm operates in continuous space and can therefore be moved in any position. Accurate roadmap visualization can only be provided if the $\mathrm{C}$-arm is positioned close enough to the exact position at which the currently displayed vessel image was acquired. To provide visual feedback regarding the current C-arm/image alignment, an "alignment signal" (Fig 1 (b)), shaped like an arrow head pointing to the left is used. It is divided in two parts: The upper half represents the alignment with respect to the Left/Right image plane, while the lower half represents the alignment with respect to the Head/Feet image plane. The degree of alignment is expressed in a color code as well as in an exact angle value. A dark blue and light blue color represents a "very good" $\left(\leq 0.5^{\circ}\right)$ and "good" $\left(\leq 1.0^{\circ}\right)$ alignment, while the color red signals an "unacceptable" $\left(>1.0^{\circ}\right)$ alignment. Roadmapping is 
only possible for a "very good" or "good" alignment. We implemented two solutions to the problem of fast and accurate C-arm alignment that appear to have their respective advantages and shortcomings and are therefore in practice commonly used interchangeably: automatic and interactive alignment.

Automatic alignment is performed in two steps, corresponding to RoRo Phases 1 and 2. First, the interventionalist uses the RoRo joystick to browse through the available projections in order to find the most suitable working view. Then, he/she clicks on the " 2 - Move-C-arm" button in the control panel (Fig. 1 (c)). This sets the C-arm system into "automatic run" mode. Simply deflecting the C-arm joystick will automatically move and stop the $\mathrm{C}$-arm at the correct position. After the position has been reached, the "Alignment signal" switches to "dark blue" and roadmapping can begin.

Interactive alignment is done in only one step. As the interventionalist moves the $\mathrm{C}$-arm back and forth along the acquisition plane, the image closest to the current $\mathrm{C}$ arm position is displayed. This is perceived as the vessel tree "following" the C-arm. In other words, browsing and aligning are done in parallel. As can be seen in Fig 2, top right, if the difference $d$ between two images is sufficiently small (e.g $1.5^{\circ}$ ), roadmapping will be possible for any $\mathrm{C}$-arm position within acquisition range. If $d$ is greater than $2.0^{\circ}$, there will be $\mathrm{C}$-arm positions, for which the alignment is "unacceptable" (red signal) and therefore roadmapping will not be allowed. In this case, the interventionalist interactively fine-tunes the $\mathrm{C}$-arm position using the alignment signal, until a "very good" or "good" alignment is reached.

While automatic alignment is exact, it requires the use of two different joysticks and is therefore relatively slow. On the other hand, interactive alignment only requires the use of the C-arm joystick but has an associated "learning curve". In most cases, images can be acquired at small enough intervals $\left(d \leq 1.5^{\circ}\right)$, in which case no finetuning is required and alignment is achieved once the working view has been found.

\subsection{Visualization}

Although visualization is limited to 2D image display, several challenges for a variety of display modes need to be addressed. For a smooth display of all guidewire manipulations, fluoroscopy frame rates of 30fps are often used. To match this frame rate, OpenGL texture mapping was employed for image display. To additionally allow for the display of non-square, non-power-of-two projections (limitation to OpenGL versions before 2.0), images are broken up into square, power-of-two tiles, which are then individually mapped to the screen. During all display modes, basic display functionality, such as window/level, zoom and pan is available. It is of particular clinical importance to allow the interventionalist to change the imaging system's zoom format or SID (source image distance) after the acquisition of the rotational run. RoRo then automatically reformats the DSA projections in real time, to reflect the changes.

Additionally, a stereo visualization mode allows the interventionalist to perceive vessel trajectories in 3D. This is achieved by simply selecting two projections as a left and right eye stereo-pair and displaying them such that each eye only sees its respective image. We implemented a stereo projection system using polarizing filters in front of two DLP projectors with matching polarized glasses and the red/blue (anaglyph) technique, using red/blue glasses. 


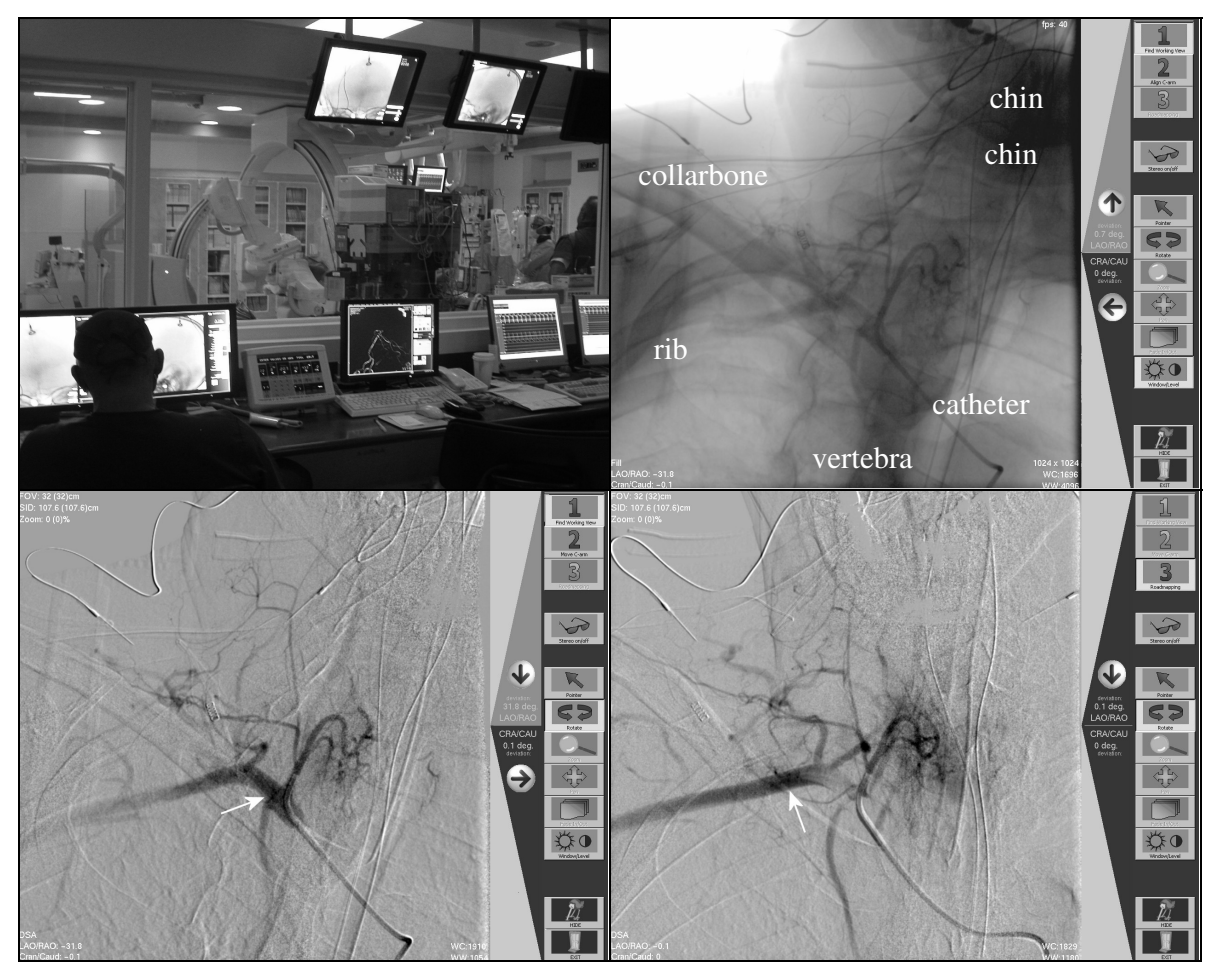

Fig. 3. Clinical results. Case \#1: Neuro interventional room (top, left). Case \#2: RoRo during Phase 1 - find working view, displaying the "rotatable roadmap" as obtained from a rotational DSA acquisition. Fill image (top, right) and subtracted images (bottom row) at different viewing angles. Note inhomogeneously opacified vessel (arrows) caused by pulsation.

During Phase 1 - find working view, the interventionalist can toggle through all types of images available. In case of a DSA acquisition (see Fig. 3) these are: native, fill and DSA run. During Phase 3 (roadmapping) three display modes are available: subtraction, anatomical background and fluoro-fade. In subtraction mode, the guidewire is segmented, by continuously subtracting the live fluoroscopic image from a previously acquired fluoro mask. The image showing the segmented wire is then subtracted from the currently selected DSA working view (see Fig. 4, left). Since subtraction increases noise, a temporal filter is used in the segmentation of the wire. The same technique is used for the anatomical background display mode (see Fig. 4, right), with the difference, that the segmented wire is subtracted from the fill image of the currently selected working view, thus showing the instrument and vessels in relation to bony structures.

However, fluoro mask creation fails in the presence of motion, in which case the fluoro-fade mode should be used. Here, the live fluoroscopic image and the current DSA working view are directly merged, by displaying them in different color channels using OpenGL color blending. 


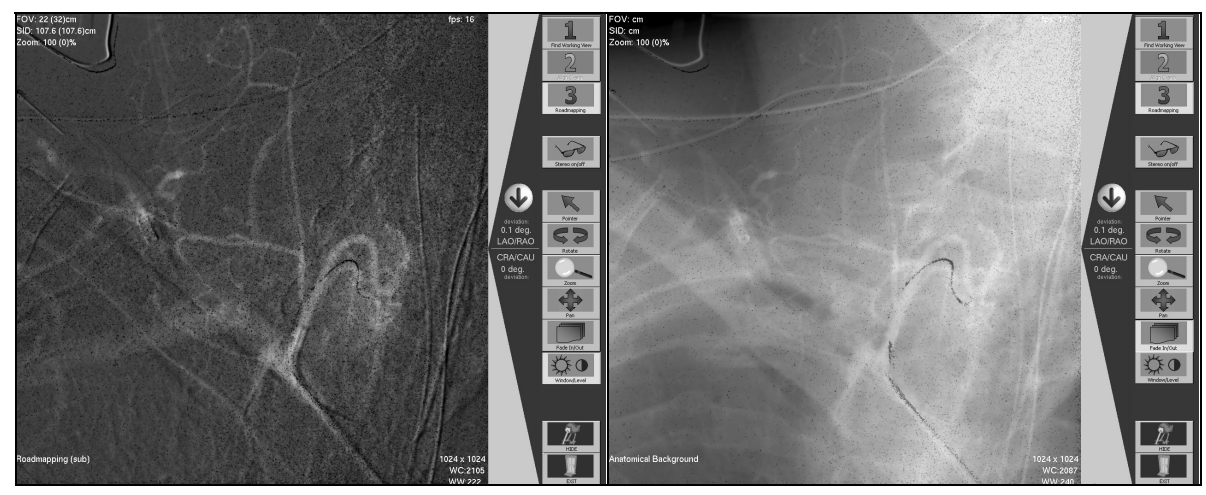

Fig. 4. RoRo Phase 3 - roadmapping. A guidewire is navigated into the feeding vessels of the tumor using subtraction mode (left) and anatomical background mode (right). Note that the alignment signal is on "blue", indicating that the C-arm is aligned with the displayed image.

\section{Clinical Results}

RoRo has been used for advanced guidewire navigation in two clinical cases. As a start, a routine and relatively straightforward case was selected. An 81 year old male presented with a cerebral aneurysm. As a routine measure, a 3D acquisition was performed to assess the neck of the aneurysm in 3D. After the treatment plan was determined, the acquired projections were sent to RoRo. A guidewire was then navigated into the aneurysm by selecting the best working view and automatically aligning the C-arm, using RoRo. However, when a $0.3 \mathrm{~mm}$ micro-guidewire was used it became evident, that for this research prototype visualization of very thin instruments needs improvement.

In the second case, a 41 year old male presented with a spinal cord tumor. The treatment plan involved embolization of the tumor to facilitate later resection. A 3D acquisition was not considered due to the proximity of the tumor to the shoulder axis. Acquisitions along the shoulder axis are typically very dark due to photon starvation and may therefore result in compromised 3D image quality. Instead, a short $82^{\circ}$ rotational acquisition (55 projections) was performed, sent to RoRo and displayed within 20s. Image quality was excellent with a pixel size of $0.2 \mathrm{~mm}$. After rotational acquisition, but before treatment started, zoom formats and SID (source image distance) were adjusted as needed. RoRo was then controlled from the tableside and repeatedly used for finding the ideal working view for guidewire navigation and embolization of the tumor's feeding vessels. Interactive C-arm alignment was found to be ideal, since fine-tuning was not required. Due to an image spacing of $1.5^{\circ}$, all $\mathrm{C}$-arm positions within the acquisition range could directly be used for roadmap navigation.

Closer examination of the angiographic images revealed another reason why a 3D acquisition and subsequent reconstruction would have been challenging. As shown by the arrows in Fig. 3, one large vessel segment was inhomogeneously opacified over time, resulting in inconsistent views, which typically creates reconstruction artifacts and compromises image quality. 


\section{Discussion and Conclusion}

RoRo effectively eliminates the main limitation of the 2D roadmapping technique, by allowing the interventionalist to visually select the best working view. In addition, RoRo provides depth perception through stereoscopic viewing, allowing the assessment of vessel trajectories in 3D. While the 3D roadmapping technique appears to be the method of choice in cases where a high quality 3D image can be acquired, in clinical practice this is a challenging task, since several factors have to be taken into account: cardiac and respiratory motion, bolus timing, filling artifacts, acquisition speed, washout rate, acquisition range and the presence of metal artifacts. Each of these factors can greatly compromise image quality and therefore the use of the image for roadmap navigation. Unfortunately, their presence and extent is often realized only after image acquisition, when the contrast material and radiation has already been administered. Until now, in such cases, the interventionalist is forced to fall back to the $2 \mathrm{D}$ roadmapping technique.

In contrast, we propose a roadmapping technique that is robust, flexible and contrast media efficient with respect to image acquisition. "Robust," because roadmapping is done directly on the $2 \mathrm{D}$ projection images without performing image reconstruction, and "flexible," because acquisition parameters can be adapted. For example, a fast $2 \mathrm{~s}$ acquisition can be performed, covering $72^{\circ}$, while the fastest $3 \mathrm{D}$ acquisition is currently $5 \mathrm{~s}$. At the same time, image quality can be optimized by performing a $3 \mathrm{~s}$ acquisition at a $2 \mathrm{k}$ matrix size and $0.15 \mathrm{~mm}$ pixel size, covering $45^{\circ}$. RoRo can be considered "contrast media efficient" since it requires an amount of contrast equivalent to the acquisition of only 2-4 conventional 2D roadmaps.

RoRo can be used directly, if a 3D acquisition is not considered, or as a fall-back method by using the projections that resulted in a reconstruction of insufficient image quality. RoRo has the potential of filling the gap between the 2D and 3D roadmapping techniques. More clinical studies are currently under way to explore applications in neuro and body imaging.

\section{References}

1. Nagel, M., Hoheisel, M., Petzold, R., Kalender, W., Krause, U.: Needle and catheter navigation using electromagnetic tracking for computer-assisted C-arm CT interventions. In: Proc. of SPIE Medical Imaging, vol. 6509 (2007)

2. Tobis, J., et al.: Digital coronary roadmapping as an aid for performing coronary angioplasty. Am. J. Cardiol. 56(4), 237-241 (1985)

3. syngo iPilot - enhanced visual information. In: Axiom Innovation in Intervention, No. 2, pp. 10-17 (March 2006), http://www.siemens.com/axiominnovation

4. Zöllei, L.: 2D-3D Rigid-Body Registration of X-Ray Fluoroscopy and CT Images. Massachusetts Institute of Technology, PhD-Thesis (August 2001)

5. Wiesent, K., Barth, K., Navab, N., Durlak, P., Brunner, T., Schuetz, O., Seissler, W.: Enhanced 3-D-reconstruction algorithm for C-arm systems suitable for interventional procedures. IEEE Trans. Med. Imaging 19, 391-403 (2000)

6. Talukdar, A.S., Wilson, D.: Modeling and Optimization of Rotational C-arm Stereoscopic X-Ray Angiography. IEEE Trans. Med. Imaging 18, 604-616 (1999) 